\title{
BMJ Open Hospital use in survivors of transient ischaemic attack compared with survivors of stroke in central China: a nested case-control study
}

\author{
Sangsang Li, ${ }^{1}$ Qingfeng Tian, ${ }^{2}$ Junxing Fan, ${ }^{3}$ Zhan Shi, ${ }^{4}$ Bingxin Guo, ${ }^{1}$ \\ Huanan Chen, ${ }^{1}$ Yapeng $\mathrm{Li}^{5}{ }^{5}$ Songhe Shi ${ }^{1}$
}

To cite: Li S, Tian Q, Fan J, et al. Hospital use in survivors of transient ischaemic attack compared with survivors of stroke in central China: a nested case-control study. BMJ Open 2019;9:e024052. doi:10.1136/ bmjopen-2018-024052

- Prepublication history and additional material for this paper are available online. To view these files, please visit the journal online (http://dx.doi. org/10.1136/bmjopen-2018024052).

Received 11 May 2018 Revised 9 April 2019

Accepted 18 June 2019

Check for updates

(C) Author(s) (or their employer(s)) 2019. Re-use permitted under CC BY-NC. No commercial re-use. See rights and permissions. Published by BMJ.

${ }^{1}$ Department of Epidemiology and Biostatistics, Zhengzhou University, Zhengzhou, China ${ }^{2}$ Department of Social Medicine, Zhengzhou University, Zhengzhou, China

${ }^{3}$ Statistical Information Center, Health and Family Planning Commission of Henan Province, Zhengzhou, China

${ }^{4}$ Department of Pharmacy, Zhengzhou People's Hospital,

Zhengzhou, UK

${ }^{5}$ Department of Neurology, The First Affiliated Hospital of Zhengzhou University, Zhengzhou, UK

Correspondence to Professor Songhe Shi; zzussh@126.com

\section{ABSTRACT}

Objectives There is a lack of knowledge regarding post-discharge hospitalisation utilisation after transient ischaemic attack (TIA) in China. The aim of this study is to quantify rehospitalisation use in survivors of TIA compared with their own previous hospital use and matched survivors of stroke.

Design Nested case-control study of electronic medical records datasets.

Setting 958 hospitals in Henan, China, from July 2012 to December 2015.

Participants In total, 4823 survivors of stroke were matched to the TIA cohort (average age: 64.5 years; proportion of men: $48.4 \%$ ) at a 1:1 ratio. All subjects with an onset of stroke/TIA were recorded with a 1-year lookback and follow-up.

Outcome measures Adjusted difference-in-differences (DID) values in 1-year hospital lengths of stay (LOSs) and readmission within 7,30 and 90 days.

Results There was an increase in hospital admissions in survivors of TIA in the year after the index hospitalisation compared with the prior year. Of the 2449 rehospitalisation events that occurred during the first year after TIA, stroke $(20.6 \%)$ was the most common reason for rehospitalisation. There was no difference in the strokespecific readmission rates between the TIA and stroke cohorts $(p=0.198)$. The TIA cohort had fewer readmissions within 30 days and 90 days after all-cause discharge compared with the controls. The corresponding covariateadjusted DID values were -3.5 percentage points $(95 \% \mathrm{Cl}-5.3$ to -1.8$)$ and $-4.5(95 \% \mathrm{Cl}-6.5$ to -2.4$)$, respectively. A similar trend was observed in the 1-year LOS. In the stratified analysis, the DID reductions were not significant in patients with more comorbidities or in rural patients.

Conclusions Compared with survivors of stroke, survivors of TIA use fewer hospital resources up to 1 year postdischarge. Greater attention to TIAs among patients with more comorbidities and rural patients may provide an opportunity to reduce hospital use.

\section{INTRODUCTION}

Readmission and length of hospital stay (LOS) are frequently used to describe the utilisation of healthcare resources. Hospital
Strengths and limitations of this study

- The strengths of this study are its large sample size and the use of difference-in-differences (DID) analysis which adjusted for trends in the transient ischaemic attack (TIA) and stroke cohorts and minimised potential confounding bias (background bias).

- A limitation of the present study was that some important and well-known risk factors, such as smoking and alcohol use, were not included in the adjusted DID model.

- We were not able to define the first-ever TIA or stroke based on the self-reported investigation.

- The quality of diagnostic codes might be influenced by variability in coding practices in hospitals located in urban and rural areas, but the concordance was excellent for all types of stroke and TIA (>95\%) after a verification of independent medical charts in $26.7 \%$ of records.

readmission significantly accounts for the high cost of inpatient care and indicates poor outcomes in the short term. ${ }^{1-3}$ Additionally, LOS is considered to be associated with the cost burden of ischaemic stroke and transient ischaemic attack (TIA). ${ }^{4}$ With the growing prominence of readmission as an outcome of interest, ${ }^{5}$ some studies report that post-discharge readmission frequently occurs with estimates varying from $9.8 \%$ to $21 \%$ at 30 days, $17 \%$ to $35 \%$ at 90 days and $31 \%$ to $55 \%$ at 1 year after acute stroke or TIA. ${ }^{26-9}$

From the perspective of the utilisation of hospitals among patients with TIA, we can provide evidence on the importance of urgent investigation and treatment of TIA. However, there is a lack of knowledge regarding post-discharge hospitalisation utilisation in patients with TIA who survive to hospital discharge in China because of the current focus on recurrent stroke after TIA. ${ }^{10}{ }^{11}$ Increasing the knowledge of hospital utilisation of patients with TIA is necessary. Unlike a stroke whose 
symptoms are obvious with rapidly developing signs of disturbance of cerebral function which often last 24 hours or longer, the symptoms of many unheeded TIAs such as weakness, numbness or visual problems may be confused with some less worrisome causes and usually last only a few minutes (and certainly no more than 24 hours). ${ }^{12}$ The risk of recurrent stroke and death after a stroke is definitely high. ${ }^{13}{ }^{14}$ Although TIA generally does not cause permanent brain damage, TIA comprises more than $40 \%$ of 30 day recurrent strokes. ${ }^{15}$ Since Chinese patients with TIA are largely unaware, undiagnosed and under treated ${ }^{16}$ knowledge of the prognosis of TIA is particularly important in China.

In addition, previous studies have inconsistent results on the comparison of the risk of adverse events (including readmission and mortality) between first-ever TIA and stroke cohorts. ${ }^{13}$ 17-19 The diverse backgrounds of the management and treatment of patients in different countries may account for the majority of this controversy. However, none of these studies were conducted in China. Furthermore, the comparison of changes in prepost hospitalisation utilisation (hospital use before and after the index hospitalisation) between TIA and stroke cohorts, which is used to understand whether the impact of TIA on subsequent hospital use is different from similar cohort and for different reasons, is also limited.

The objectives of this study were to evaluate subsequent hospital use of survivors of TIA compared with their own previous hospital use, which was useful to assess the extent to which changes were associated with TIA, as well as to examine the pre-post changes among survivors of TIA relative to those in matched patients with stroke.

\section{METHODS}

\section{Study design and population}

This nested case-control study was conducted based on data from the electronic medical record (EMR) database in the Henan Province, China. In total, 958 hospitals involved in acute stroke care contributed, during the period from July 2012 to December 2015. This study was exempted by the ethics committee of Zhengzhou University in China. In this study, patients aged $\geq 20$ years who had been admitted with a first-ever acute stroke (cerebral haemorrhage, cerebral infarction or subarachnoid haemorrhage) or TIA to the hospitals and subsequently survived to hospital discharge were included. The stroke cohort was regarded as the control group. The index stroke/TIA hospitalisation occurred between 21 August 2013 and 31 December 2014, which was defined as a firstever TIA or a stroke without a history of cerebrovascular disease based on previous EMR baseline data (using a 1-year to 2-year look-back before the index hospitalisation) that was linked to medical insurance datasets. The index hospitalisation of a TIA (G45, excluding G45.4) or acute stroke (I60, I61, I63, I64) was defined using the International Classification of Diseases Tenth Revision (ICD-10) codes with regular application. ${ }^{20} 21$ The length of observation started 1 year before the index hospital admission to 1 year following the index discharge date.

\section{Outcomes}

The outcomes of interest were hospital use before and after the index hospitalisation: (1) 1-year LOS before the index hospitalisation or after the index discharge, (2) readmission within 7,30 and 90 days after an all-cause discharge in the year prior to and after the index hospitalisation and (3) readmission for stroke or all causes within 7, 30, 90 and 365 days after index discharge during the 12-month follow-up period. LOS for a hospital admission was measured in days as the difference between the date of admission and the date of discharge. Furthermore, considering that the study population was fixed to the baseline hospitalisation before the index hospitalisation (even though many of them would not be readmitted in the subsequent follow-up), the 1-year LOS was calculated using the 1-year prior-index or post-index sum of all single LOSs for patients with readmissions in the year after the index discharge. The LOS of the index hospitalisation was not included in the 1-year LOS. Therefore, the 1-year LOS was the excess LOS before/after the index hospitalisation. For the second type of outcome, we used the readmission after an all-cause discharge to measure the simple increase (simple pre-post analysis) in hospital service use before and after the onset of stroke/TIA as well as to evaluate the adjusted changes of increases (DID analysis) between the stroke and TIA cohorts.

\section{Matching of TIA and stroke hospitalisations}

A coarsened exact matching approach was implemented because, while retaining a large sample size, it has useful advantages relating to the propensity score matching method and achieves the best possible covariate balance without making unverifiable assumptions on the data-generation process. ${ }^{22}{ }^{23}$ Stroke baseline hospitalisations were matched to the baseline TIA hospitalisation at a 1:1 ratio on eight confounding indicators including age, sex, living areas (urban and rural), marital status (married, never married or widowed/divorced), Charlson Comorbidity Index (CCI), surgery (yes or no), LOS and type of health insurance (system for urban employees, system for urban residents, rural cooperative system, other systems), without replacement. CCI quantifying 19 conditions or complications as a prognostic marker for poor outcomes was calculated by the updated coding algorithms. ${ }^{24}$ The CCI was matched with a classification of scores of 0,1 to 2 and $3+$. The dichotomous variables, including sex, living area and surgery, were matched exactly. In terms of LOS, lengths of $1,2,3,4,5,6,7$ and 8 to 30,31 to 60 and $61+$ days were chosen as the matching classifications. ${ }^{25}$ For age, health insurance and marital status, we used the default automatic binning algorithm. ${ }^{26}$

\section{Statistical analysis}

Continuous variables were described with means (SDs) or medians (IQRs), and categorical variables were described 
with frequencies (percentages). To compare differences between cohorts, the $\chi^{2}$ test was used for categorical variables, and the Wilcoxon-Mann-Whitney rank-sum test was used for continuous variables.

After identifying the stroke subgroup with cerebral infarction (I63), haemorrhage (I60 or I61) and others that were not specified as either a haemorrhage or a cerebral infarction (I64), we constructed Nelson-Aalen cumulative event curves, with the Kaplan-Meier method, to demonstrate the timing of the first all-cause and stroke readmission after the index discharge.

The difference-in-differences (DID) analysis was a quasi-experimental technique, ${ }^{27}$ which was used to compare the changes over time in hospital utilisation between the TIA and stroke cohorts. It was assumed that the common trends of hospital care needs before the index hospitalisation among TIA and stroke cohorts were the same and we measured the changes at 1 year following the index discharge relative to the year prior to the index hospitalisation. ${ }^{28}$ The DID estimate was calculated by subtracting the 'post' (follow-up) outcome from the 'pre' (look-back) result in the TIA and stroke cohorts ${ }^{28}$ :

DID=difference ${ }_{\mathrm{TIA}}-$ difference $_{\text {stroke }}$

A negative value of the DID estimate represents less absolute hospital needs for survivors of TIA than survivors of stroke. Covariate-adjusted estimates of DID were adjusted using employment status (employed, unemployed and other), as well as the eight other matching variables mentioned earlier. By fitting a DID model in population-based data with the subtraction of background changes, we can generate estimates of the independent effects of stroke/TIA.

Two-tailed and $\mathrm{p}$ values less than 0.05 were regarded as statistically significant. We also performed stratified analyses based on baseline indicators of age group ( $\geq 65$ or $<65$ ), CCI $(\geq 3$ or $<3$ ) and living areas (urban and rural). All statistical analyses were performed using Stata V.11 (StataCorp).

Because potential temporal changes in hospitalisation use and in-hospital mortality might affect the DID estimates, a sensitivity analysis was performed by rerunning the one-to-one match by quarters of a year, in addition to the eight other matching variables.

\section{Patient and public involvement}

Patients and/or the public were not directly involved in the design, recruitment or implementation of the study.

\section{RESULTS}

\section{Characteristics}

In total, 26505 survivors of a first-ever stroke/TIA met the inclusion criteria. After a 1:1 matching based on age, sex and other covariates, 4823 TIA cases and 4823 matched patients with stroke were included in the subsequent analysis. The final ascertainment of the study population, with detailed inclusion and exclusion criteria, is summarised in figure 1.
Table 1 shows the characteristics of the study subjects in the year before and after the index hospitalisation. For the baseline characteristics, the mean age was $64.5 \pm 12.6$ years old, $51.6 \%$ of the subjects were women and the median comorbidity burden was 1.0 (IQR 0.0-1.0). As expected, the eight matched characteristics did not differ significantly between TIA and stroke cohorts in the baseline comparison. For the follow-up characteristics in the final record after the index discharge, the median LOS was 10 days (IQR 7-14) and 11 days (IQR 7-16) for the TIA and stroke cohorts $(\mathrm{p}<0.01)$, respectively, and the surgery rate differed between the survivors of TIA and the controls ( $5.0 \%$ vs $9.1 \%$, respectively). Similarly, there were statistically significant differences in the CCI and health insurance between the two groups after the index discharge.

\section{Hospital use in the first year after discharge for stroke/TIA}

Hospital use for the overall population in the next year after the index hospitalisation is shown in online supplementary table 1 . Recurrent stroke rates within 30 days and 90 days were not significantly different between the two cohorts. The 1-year first-readmission rate for survivors of TIA was less frequent than that in the stroke cohort $(\mathrm{p}<0.01)$. The overall in-hospital mortality was $1.0 \%$ within 1 year. Patients may have $>1$ rehospitalisation event, resulting in 5649 readmissions during the 1-year follow-up (see online supplementary table 2 ). There were 2479 and 3170 readmission events for the TIA and stroke cohorts, respectively. The three major causes for 1-year readmissions were recurrent stroke $(21.4 \%)$, respiratory infection $(17.8 \%)$ and coronary artery disease $(17.0 \%)$. The difference in readmission rates for stroke between the TIA $(20.6 \%)$ and stroke $(22.0 \%)$ cohorts was not significant $(\mathrm{p}=0.198)$. The 1 -year readmission rate after index discharge was markedly higher for the subgroups of patients with more comorbidities (CCI $\geq 3$ ) as well as patients living in urban areas (see online supplementary tables 3 and 4 ).

Figure 2 shows the unadjusted risk of all-cause and stroke readmissions according to stroke subtype and TIA. Patients with cerebral ischaemic stroke had a significantly higher risk of readmission for all causes within 1 year compared with patients with TIA as the index hospitalisation $(\mathrm{p}<0.05)$. The 1-year Kaplan-Meier estimates of stroke rehospitalisation were not significant between the groups $(\mathrm{p}=0.06)$.

\section{Hospital services use before and after the index hospitalisation}

In the simple pre-post analyses, there were apparent increases for both the TIA and stroke cohorts in the year after the index hospitalisation (figure 3). For 90-day readmission after an all-cause discharge, the TIA and stroke cohorts markedly increased by 3.9 percentage points (95\% CI 2.5 to 5.3 ) and 9.0 percentage points (95\% CI 7.5 to 10.6), respectively. It was also notable that the increase in the 1-year LOS in the two cohorts was significant. 


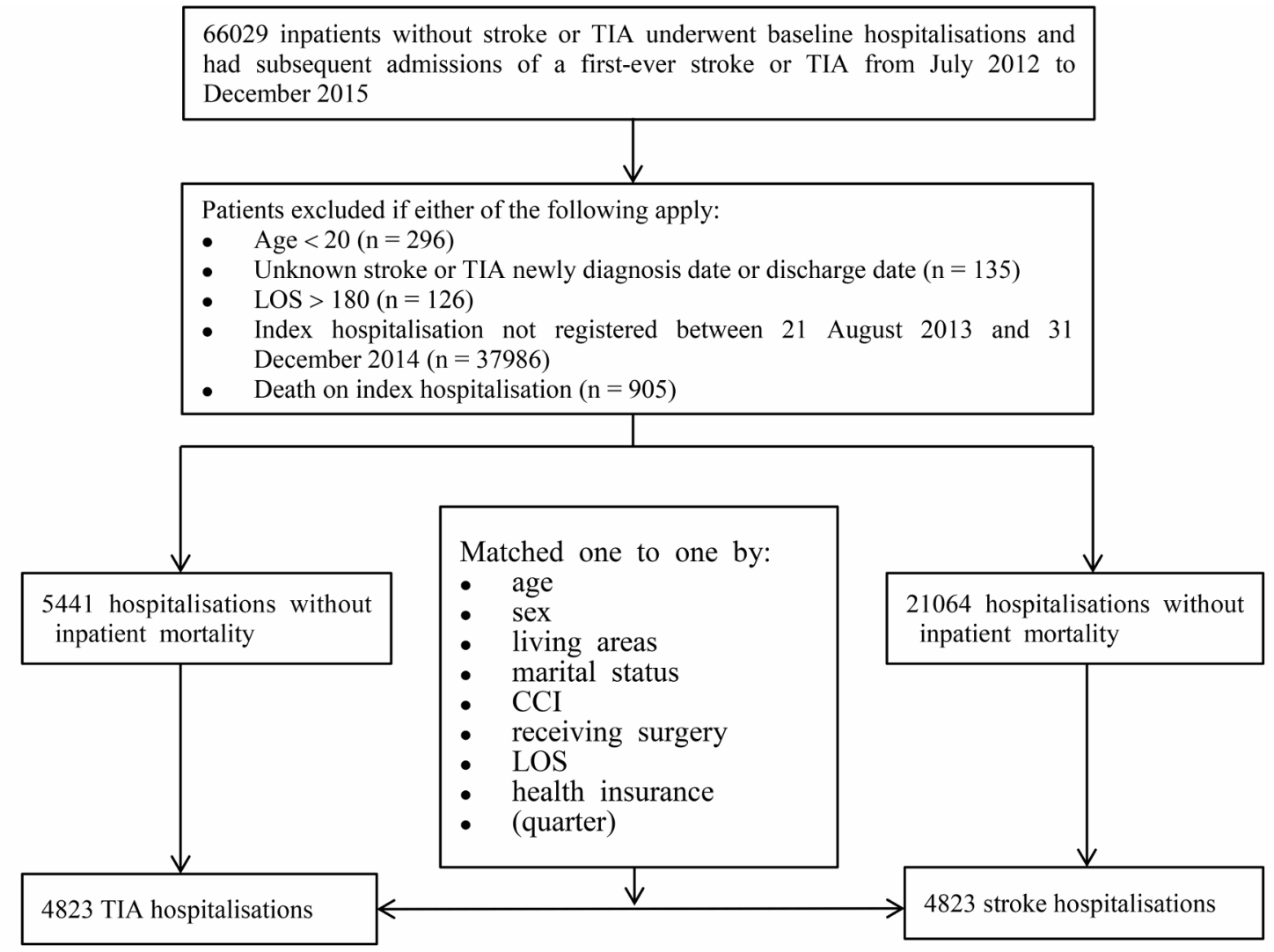

Figure 1 Flow chart for the identification and matching of the TIA and stroke cohorts. CCI, Charlson Comorbidity Index; LOS, hospital length of stay; TIA, transient ischaemic attack.

Patients aged $\geq 65$ years in both groups had a large degree of increased hospital use.

\section{Changes in hospital use after TIA versus stroke hospitalisations}

The DID estimates of readmissions after an all-cause discharge and the 1-year LOS are shown in figure 3. There was a significantly smaller increase in the adjusted 1-year LOS of -2.7 days (95\% CI -5.3 to -0.2 ) in the TIA cohort compared with the stroke cohort. Patients with TIA had a smaller increase in the adjusted 30 day readmission rate compared with the stroke cohort by -3.5 percentage points (95\% CI -5.3 to -1.8$)$. There was also a smaller augmentation of -4.5 (95\% CI -6.5 to -2.4$)$ in the adjusted 90-day readmission rate in patients with TIA compared with patients with stroke. A sensitivity analysis, using quarters of a year in addition to the other eight covariates to match 3637 index hospitalisations, demonstrated analogous tendencies (figure 3).

In most subgroup analyses, the DID reductions of 30-day and 90-day readmission were significant except for patients with a CCI $\geq 3$ and patients living in rural areas. It was obvious that absolute values from the adjusted DID were high for readmissions in urban patients within 7 , 30 and 90 days after an all-cause discharge. The corresponding values were -2.4 percentage points $(95 \% \mathrm{CI}$ -4.0 to -0.7$),-4.6$ percentage points $(95 \%$ CI -6.9 to -2.4 ) and -5.3 percentage points $(95 \% \mathrm{CI}-7.9$ to -2.6$)$, respectively. Similar large tendencies towards readmission and LOS were observed in patients with a CCI $<3$.

\section{DISCUSSION}

Based on the large retrospective data, this study had several key findings. First, hospital use was more frequent in the stroke cohort than in the TIA cohort in the first year after the index hospitalisation. Second, recurrent stroke was the most common reason for rehospitalisation of the TIA cohort during the follow-up year. There were no differences in the causes of readmission in either group. Third, the hospital utilisation after the index TIA hospitalisation was significantly higher compared with the previous hospital resources used. Finally, for the overall population, patients with TIA experienced a significantly smaller increase in hospital use compared with patients with stroke. Nevertheless, there were some discrepant results from different stratified analyses which offered more details for the increased hospital use among patients with TIA.

Regarding 1-year readmission following the index discharge, stroke was the most common reason for rehospitalisation which was identical to other reports. ${ }^{1829}$ Moreover, $36.0 \%$ of stroke patients were readmitted in the first year, which was comparable to studies from other areas where 1-year readmission rates varied from $31 \%$ to $50 \%$ 
Table 1 Characteristics over 2 years for the matched survivors of TIA and stroke

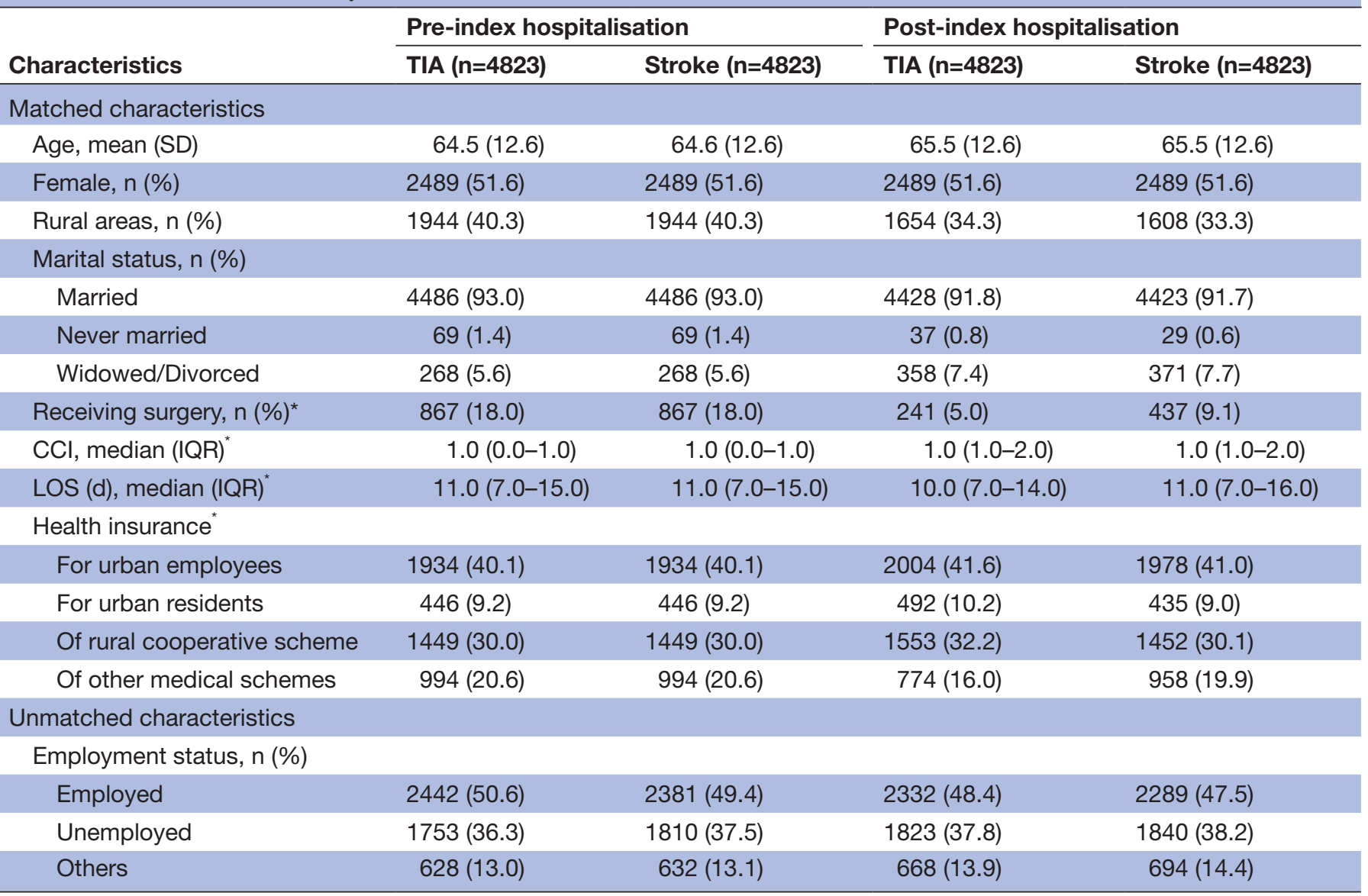

${ }^{*} \mathrm{P}<0.05$ ( $\chi^{2}$ test or Wilcoxon-Mann-Whitney rank-sum test) for matched survivors of TIA and stroke in the period after the index hospitalisation.

CCI, Charlson Comorbidity Index; IQR, interquartile range; LOS, length of stay; TIA, transient ischaemic attack.

after stroke. ${ }^{2}{ }^{30-32}$ However, $30.1 \%$ of patients with TIA were readmitted within the first year which was lower than a previous report $(48.3 \%) .{ }^{9}$ This result was mainly caused by the large number of elderly subjects in that study, while there was a large proportion $(50.0 \%)$ of patients with TIA aged $<65$ years in our study.

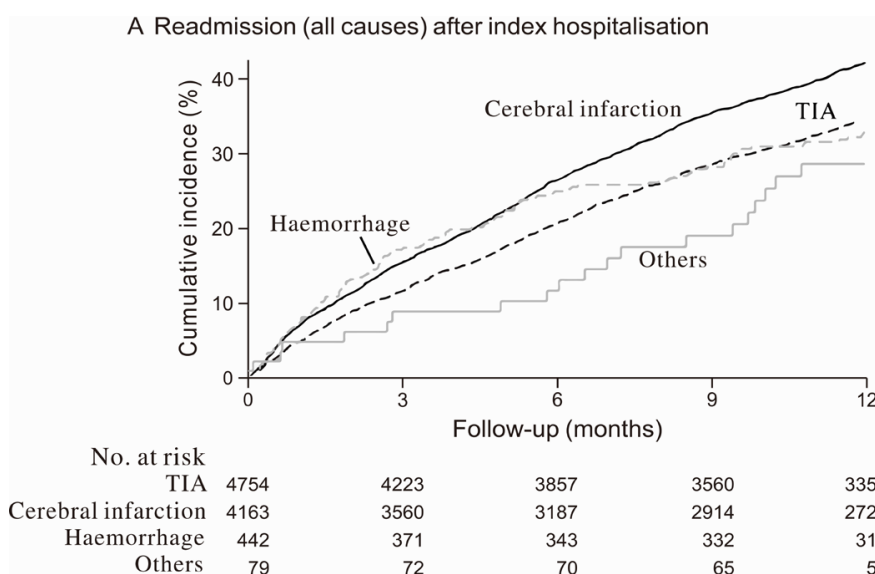

In addition, patients with TIA were less likely to be readmitted within 1 year after the index discharge compared with patients with stroke which is contrary to a previously published report. ${ }^{18}$ The setting in different countries (Australia and China) may account for this difference. Compared with this previous report, the key strengths of

B Stroke readmission after index hospitalisation

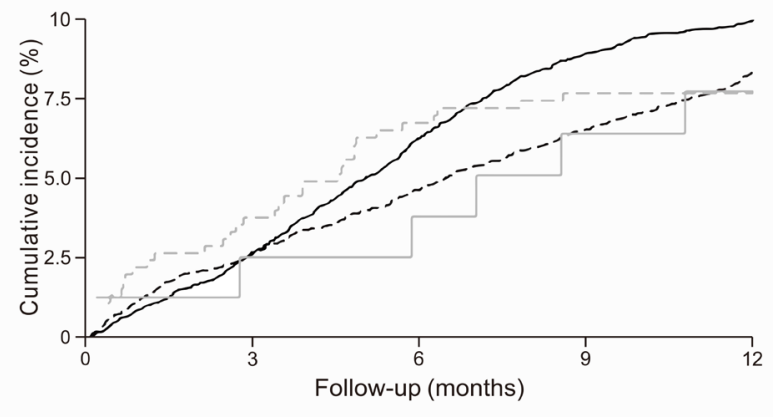

$\begin{array}{rrrrr}4811 & 4684 & 4593 & 4506 & 4425 \\ 4267 & 4154 & 4007 & 3901 & 3861 \\ 459 & 442 & 429 & 425 & 425 \\ 80 & 78 & 77 & 75 & 74\end{array}$

Figure 2 Nelson-Aalen cumulative incidence curves. (A) First readmission (all causes) within 1 year after discharge from the index hospitalisation. (B) Stroke readmission within 1 year after discharge from the index hospitalisation. TIA, transient ischaemic attack. 


\begin{tabular}{|c|c|c|c|c|c|}
\hline & \multicolumn{2}{|c|}{ Increase $(95 \% \mathrm{CI})$} & \multirow{2}{*}{$\begin{array}{l}\text { Unadjusted DID } \\
\text { Estimate (95\%CI) }\end{array}$} & \multicolumn{2}{|c|}{ Covariates-adjusted DID } \\
\hline & TIA & Stroke & & & Estimate $(95 \% \mathrm{CI})$ \\
\hline \multicolumn{6}{|l|}{ Overall population } \\
\hline Readmission within 7 days (\%) & $1.8(0.9,2.6)$ & $3.4(2.5,4.4)$ & $-1.7(-3.0,-0.4)$ & - & $-1.2(-2.5,0.1)$ \\
\hline Readmission within 30 days(\%) & $1.7(0.6,2.9)$ & $6.0(4.7,7.2)$ & $-4.2(-6.0,-2.5)$ & - & $-3.5(-5.3,-1.8)$ \\
\hline Readmission within 90 days(\%) & $3.9(2.5,5.3)$ & $9.0(7.5,10.6)$ & $-5.1(-7.2,-3.1)$ & - & $-4.5(-6.5,-2.4)$ \\
\hline 1-Year LOS (days) & $2.8(1.0,4.6)$ & $6.4(4.5,8.2)$ & $-3.5(-6.1,-1.0)$ & - & $-2.7(-5.3,-0.2)$ \\
\hline \multicolumn{6}{|l|}{ Patients aged $<65$ years } \\
\hline Readmission within 7 days (\%) & $0.8(-0.3,2.0)$ & $3.1(1.7,4.4)$ & $-2.3(-4.0,-0.5)$ & - & $-1.9(-3.6,-0.1)$ \\
\hline Readmission within 30 days (\%) & $0.5(-1.1,2.1)$ & $4.7(3.0,6.5)$ & $-4.3(-6.7,-1.9)$ & - & $-3.5(-5.9,-1.1)$ \\
\hline Readmission within 90 days $(\%)$ & $2.3(0.4,4.2)$ & $7.3(5.2,9.3)$ & $-5.0(-7.8,-2.2)$ & - & $-4.2(-7.0,-1.4)$ \\
\hline 1-Year LOS (days) & $0.7(-1.5,2.9)$ & $4.6(1.9,7.2)$ & $-3.9(-7.3,-0.4)$ & - & $-3.0(-6.4,0.4)$ \\
\hline \multicolumn{6}{|l|}{ Patients aged $\geq 65$ years } \\
\hline Readmission within 7 days (\%) & $2.7(1.5,4.0)$ & $3.8(2.4,5.2)$ & $-1.0(-2.9,0.8)$ & 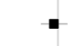 & $-0.6(-2.4,1.3)$ \\
\hline Readmission within 30 days $(\%)$ & $3.0(1.3,4.8)$ & $7.1(5.3,9.0)$ & $-4.1(-6.7,-1.5)$ & - & $-3.5(-6.0,-1.0)$ \\
\hline Readmission within 90 days $(\%)$ & $5.5(3.4,7.6)$ & $10.7(8.6,12.9)$ & $-5.2(-8.3,-2.2)$ & - & $-4.6(-7.7,-1.6)$ \\
\hline 1-Year LOS (days) & $4.5(1.8,7.2)$ & $7.8(5.2,10.3)$ & $-3.3(-7.0,0.5)$ & - & $-2.6(-6.2,1.1)$ \\
\hline \multicolumn{6}{|l|}{ Patients living in urban areas } \\
\hline Readmission within 7 days (\%) & $1.7(0.6,2.8)$ & $4.7(3.4,5.9)$ & $-3.0(-4.7,-1.3)$ & - & $-2.4(-4.0,-0.7)$ \\
\hline Readmission within 30 days $(\%)$ & $2.1(0.6,3.6)$ & $7.5(5.9,9.2)$ & $-5.4(-7.7,-3.2)$ & - & $-4.6(-6.9,-2.4)$ \\
\hline Readmission within 90 days $(\%)$ & $4.4(2.5,6.2)$ & $10.5(8.5,12.4)$ & $-6.1(-8.8,-3.4)$ & -- & $-5.3(-7.9,-2.6)$ \\
\hline 1-Year LOS (days) & $3.6(1.2,6.0)$ & $7.5(5.2,9.8)$ & $-3.9(-7.2,-0.6)$ & - & $-3.0(-6.3,0.3)$ \\
\hline \multicolumn{6}{|l|}{ Patients living in rural areas } \\
\hline Readmission within 7 days (\%) & $1.8(0.5,3.2)$ & $0.9(-0.5,2.4)$ & $0.9(-1.1,2.9)$ & & $0.9(-1.1,2.9)$ \\
\hline Readmission within 30 days (\%) & $1.0(-0.9,2.9)$ & $2.7(0.7,4.7)$ & $-1.7(-4.4,1.1)$ & - & $-1.4(-4.1,1.4)$ \\
\hline Readmission within 90 days $(\%)$ & $2.8(0.5,5.1)$ & $5.8(3.5,8.2)$ & $-3.0(-6.3,0.2)$ & $\cdot$ & $-2.8(-6.1,0.4)$ \\
\hline 1-Year LOS (days) & $0.6(-1.9,3.0)$ & $2.6(0.0,5.1)$ & $-2.0(-5.5,1.5)$ & 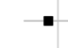 & $-1.4(-4.9,2.0)$ \\
\hline \multicolumn{6}{|l|}{ Inpatients with $\mathrm{CCl}<3$} \\
\hline Readmission within 7 days (\%) & $1.8(0.9,2.7)$ & $3.5(2.6,4.5)$ & $-1.7(-3.1,-0.4)$ & - & $-1.6(-2.9,-0.3)$ \\
\hline Readmission within 30 days $(\%)$ & $1.7(0.5,2.9)$ & $5.7(4.4,7.0)$ & $-4.0(-5.8,-2.2)$ & - & $-3.8(-5.6,-2.0)$ \\
\hline Readmission within 90 days $(\%)$ & $3.8(2.3,5.2)$ & $8.9(7.4,10.4)$ & $-5.1(-7.2,-3.0)$ & - & $-4.9(-7.0,-2.8)$ \\
\hline 1-Year LOS (days) & $2.9(1.0,4.7)$ & $6.3(4.4,8.2)$ & $-3.5(-6.1,-0.8)$ & - & $-3.5(-6.1,-0.8)$ \\
\hline \multicolumn{6}{|l|}{ Inpatients with $\mathrm{CCl} \geq 3$} \\
\hline Readmission within 7 days (\%) & $1.4(-2.8,5.7)$ & $1.8(-3.2,6.8)$ & $-0.4(-6.9,6.2)$ & + & $-0.6(-7.1,5.9)$ \\
\hline Readmission within 30 days $(\%)$ & $2.2(-3.5,7.9)$ & $10.2(3.4,17.0)$ & $-8.0(-16.9,0.9)$ & & $-8.2(-17.0,0.6)$ \\
\hline Readmission within 90 days $(\%)$ & $5.8(-1.0,12.6)$ & $11.3(3.7,18.9)$ & $-5.5(-15.7,4.7)$ & $\cdot$ & $-5.8(-15.9,4.4)$ \\
\hline 1-Year LOS (days) & $2.3(-4.7,9.3)$ & $6.7(-0.6,14.0)$ & $-4.4(-14.5,5.7)$ & - & $-5.5(-15.5,4.5)$ \\
\hline \multicolumn{6}{|l|}{ Quarter-matched pairs } \\
\hline Readmission within 7 days (\%) & $1.7(0.7,2.7)$ & $4.5(3.4,5.6)$ & $-2.8(-4.3,-1.4)$ & $=$ & $-2.5(-3.9,-1.1)$ \\
\hline Readmission within 30 days $(\%)$ & $1.7(0.3,3.1)$ & $6.3(4.9,7.8)$ & $-4.6(-6.7,-2.6)$ & - & $-4.0(-6.0,-2.0)$ \\
\hline Readmission within 90 days $(\%)$ & $4.0(2.4,5.7)$ & $8.8(7.1,10.5)$ & $-4.8(-7.2,-2.4)$ & - & $-4.3(-6.6,-2.1)$ \\
\hline 1-Year LOS (days) & $2.1(0.2,4.0)$ & $5.1(3.0,7.2)$ & $-3.0(-5.9,-0.2)$ & - & $-2.9(-5.7,-0.1)$ \\
\hline
\end{tabular}

Figure 3 Unadjusted and covariate-adjusted DID estimates comparing changes in hospital use between the TIA and stroke cohorts. Covariate-adjusted DID was adjusted for age, sex, area, marital status, CCI, surgery, LOS, health insurance and employment status. Readmissions indicate patients who were readmitted to hospitals after an all-cause discharge. CCl, Charlson Comorbidity Index; DID, difference-in-differences; LOS, hospital length of stay; TIA, transient ischaemic attack.

our analysis include a larger sample size and the use of a DID analysis adjusting for trends in the control group which minimises potential confounding bias (background bias) ${ }^{33}$ To our knowledge, studies on the comparison of pre-post hospitalisation utilisation between stroke and TIA cohorts are sparse, and there are no other studies with similar analyses exploring within-person changes for patients admitted for TIA.

Currently, there is a lack of knowledge regarding the rehospitalisation use of survivors of TIA in China. ${ }^{11}$ In this respect, the societal benefit of our findings is to increase the knowledge and awareness of hospital use for patients with TIA. We found that a TIA independently influenced hospital use from the simple pre-post analysis. Moreover, from the reductions of the adjusted DID estimates, we confirmed that if a patient with TIA sought medical attention, he/she had a reduction of $4.5 \%$ for 90 day hospital use and a reduction of 2.7 days spent in hospitals during the follow-up period compared with a patient with stroke. In other words, patients with TIA receiving the treatment after seeking medical attention have the benefit of a lesser need for rehospitalisation than the matched stroke 
cohort. It is noteworthy that the treatment recommendations for a patient admitted for TIA generally include: antiplatelet therapy, statins, anticoagulation as required and medication for controlling other conditions such as high blood pressure and diabetes. Antiplatelet therapy is the key intervention to reduce the risk of early recurrent stroke and this kind of secondary prevention within the first hours and days after a TIA or minor stroke seems to reduce the risk of recurrent stroke by as much as $80 \% .^{34}$ Additionally, changes in lifestyle behaviours, such as smoking cessation and weight reduction, are also important. When improving the strategies of prevention to reduce unnecessary rehospitalisations, such as a closer follow-up in primary care, public policy-makers should pay more attention to TIAs among patients living in rural areas and patients with more comorbidities based on the results of stratified analysis. In addition to these strategies of secondary prevention, the importance of public education should be highlighted because patients frequently fail to recognise or act on TIA symptoms with confusion and attribution to other (less worrisome) causes and either delay seeking medical attention or do not seek medical attention at all. ${ }^{1236}$ Based on a previous investigation, ${ }^{16}$ only $3.08 \%$ of Chinese adults had any knowledge about TIA and a conservative estimate of the number of Chinese TIA cases who were not admitted to hospitals was approximately 22.7 million people in 2010 . Nonetheless, there is a lack of effective methods to improve the public sensitivity of patients with TIA to seek medical attention in a timely manner. At present, the basis of Chinese public education is formed by the Face, Arm, Speech, Time test which is effective at improving the recognition of stroke but not TIA. ${ }^{12}$ Public education for TIA recognition is urgent.

In the stratified analysis of areas, what may contribute to the non-significance of the estimated DID values is that adults from rural areas in China are less sensitive to early symptoms of stroke/TIA and are less likely to use inpatient services than their urban counterparts. ${ }^{37}$ Our study also found that 1 year readmission rates $(26.6 \%$ vs $32.4 \%$ ) and LOS (median 11 vs 13) after the index TIA discharge in rural patients were lower than in urban citizens, respectively, which differed from previous findings that the utilisation of hospitals in patients living in rural areas was larger than in patients living in urban areas. ${ }^{16}$ It is possible that, although rural residents often have poor adherence to treatments and are unaware of risk factors, they are influenced by accelerating urbanisation and the improvement of the Chinese healthcare system in recent years. ${ }^{38}$ Subsequently, rural patients have better access to hospital care, indicating that they are able to seek medical attention from high-level hospital units similar to urban citizens. ${ }^{40}$ Nevertheless, combined with the non-significance of the DID estimates in rural survivors, policy-makers still need to focus on improving public awareness of suspected TIAs among rural patients.

A previous study reported that inpatients with a stroke or TIA with a greater number of comorbidities were associated with readmission within 30 days and 1 year after discharge, ${ }^{18}$ which is consistent with our finding that patients with higher CCI scores $(\geq 3)$ were more likely to be readmitted during the follow-up period (see online supplementary table 4 ). Meanwhile, a CCI $<3$ was associated with a reduced need for hospital care in the TIA cohort compared with the stroke cohort. There was, however, no significant difference in the adjusted DID estimates in patients with a CCI $\geq 3$. The severe conditions requiring much hospital use both before and after the index hospitalisation in the two cohorts might account for this finding. Non-significant reductions in the DID estimates combined with larger simple prepost outcomes for people with a CCI $\geq 3$ suggest a need for greater attention to TIAs among patients with more comorbidities to reduce hospital use.

There are several limitations to the present study. First, this study did not include socioeconomic characteristics, lifestyle factors and clinical indicators indicative of the severity of the illness or the functional status. Additionally, other types of healthcare resource use, such as medical visits in the community, were not included, and the assessment of self-reported health status, which was important for survivors of stroke/TIA and their families during the follow-up period, was unable to be considered, ${ }^{41}$ because our data could only be extracted from medical records during the hospital stay. For the severity of the illness, the CCI, a proxy parameter, was used to alleviate the influence of the absence of some factors that might affect outcomes of stroke/TIA. Second, all the subjects discharged alive were chosen with baseline records on previous hospitalisations to confirm their index hospitalisation of a first-ever TIA or stroke. This method may lead to an overestimation of readmissions after the index discharge because hospitalisations were shown to increase the 30-day and 90-day readmission rates after discharge in a previous report. ${ }^{42}{ }^{43}$ Since health insurance covered the overwhelming majority of the Chinese population, EMRs were linked to the medical insurance data to ensure that previous nationwide hospital admissions were recorded. Third, the use of retrospective data from a single province may limit the generalisability to the rest of China. However, Henan Province, situated at the centre of China is the most populous province in China and serves approximately 100 million people per year. The utilisation of medical resources in Henan could reflect the real situation of central China to a large degree. Finally, ICD coding quality may be contaminated by variability in coding practices in hospitals located in urban and rural areas. ${ }^{1844}$ To verify the quality of appropriate codes and reduce this possible bias, we randomly audited 2572 medical charts. The diagnostic concordance was up to $99.0 \%$ and $91.9 \%$ in urban and rural hospitals, respectively. In addition, teaching hospitals are responsible for training health records technologists from urban and rural hospitals in Henan each year. Even so, further study on assessing regional variability from hospital performance needs to be explored. ${ }^{45}$ 


\section{CONCLUSION}

TIA is a sufficient cause for an increase in hospital utilisation. Moreover, there was a smaller increase in hospital use in survivors of TIA compared with the stroke cohort. This smaller increase is obvious in subgroups of patients with fewer comorbidities and patients living in urban areas. From the perspective of hospital use, individuals with transient neurological symptoms for which they seek medical attention have a better prognosis than patients who had a stroke. Additionally, policy-makers may target more interventions to reduce unnecessary rehospitalisations, such as closer follow-up in primary care and the improvement of the public awareness of TIA among individuals living in rural areas and individuals with more comorbidities.

Acknowledgements The authors are grateful to Qing Cai, Niao Wang for help on verification of International Classification of Diseases (ICD-10) coding. In addition, they thank Dr Fatiha Karam and Professor Ling Wang for support of English paper proofreading.

Contributors SL, QT, JF and SS designed the study and drafted the manuscript. SL, SS and JF participated in the data collection and analysis. SL, SS, ZS, BG, HC and $\mathrm{YL}$ interpreted the data and reviewed the manuscript. All authors read and approved the final version of the manuscript.

Funding This study was supported by the National Key Research and Development Program (grant numbers: 2017YFC1307705) and Science and Technology Development Program of Zhengzhou (grant number: 141PPTGG441).

Competing interests None declared.

Patient consent for publication Next of kin consent obtained.

Ethics approval As this study used existing and anonymised data, an ethical review was exempted from the ethics committee of Zhengzhou University. None of the authors were involved in anonymizing the data. Approvals for the use of anonymised EMR data were obtained from the Health and Family Planning Commission of Henan Province.

Provenance and peer review Not commissioned; externally peer reviewed.

Data sharing statement All investigators should sign an agreement that guarantees patient confidentiality before using the data. All data relevant to the given manuscript are available and can be accessed by contacting SS (zzussh@ 126.com).

Open access This is an open access article distributed in accordance with the Creative Commons Attribution Non Commercial (CC BY-NC 4.0) license, which permits others to distribute, remix, adapt, build upon this work non-commercially, and license their derivative works on different terms, provided the original work is properly cited, appropriate credit is given, any changes made indicated, and the use is non-commercial. See: http://creativecommons.org/licenses/by-nc/4.0/.

\section{REFERENCES}

1. Jencks SF, Williams MV, Coleman EA. Rehospitalizations among patients in the medicare fee-for-service program. $N$ Engl $J$ Med 2009;360:1418-28.

2. Lin HJ, Chang WL, Tseng MC. Readmission after stroke in a hospital-based registry: risk, etiologies, and risk factors. Neurology 2011;76:438-43.

3. Zuckerman RB, Sheingold SH, Orav EJ, et al. Readmissions, observation, and the hospital readmissions reduction program. $N$ Engl J Med 2016;374:1543-51.

4. Buisman LR, Tan SS, Nederkoorn PJ, et al. Hospital costs of ischemic stroke and TIA in the Netherlands. Neurology 2015;84:2208-15.

5. Axon RN, Williams MV. Hospital readmission as an accountability measure. JAMA 2011;305:504-5.

6. Bjerkreim AT, Thomassen L, Brøgger J, et al. Causes and predictors for hospital readmission after ischemic stroke. J Stroke Cerebrovasc 2015;24:2095-101.
7. Bravata DM, Ho SY, Meehan TP, et al. Readmission and death after hospitalization for acute ischemic stroke: 5-year follow-up in the medicare population. Stroke 2007;38:1899-904.

8. Fehnel CR, Lee Y, Wendell LC, et al. Post-acute care data for predicting readmission after ischemic stroke: A nationwide cohort analysis using the minimum data set. J Am Heart Assoc 2015;4:e002145.

9. Lichtman JH, Jones SB, Watanabe E, et al. Elderly women have lower rates of stroke, cardiovascular events, and mortality after hospitalization for transient ischemic attack. Stroke 2009;40:2116-22.

10. von Weitzel-Mudersbach $\mathrm{P}$, Andersen G, Hundborg $\mathrm{HH}$, et al. Transient ischemic attack and minor stroke are the most common manifestations of acute cerebrovascular disease: a prospective, population-based study - the Aarhus TIA study. Neuroepidemiology 2013;40:50-5.

11. Wu L, Wang A, Wang X, et al. Factors for short-term outcomes in patients with a minor stroke: results from China National Stroke Registry. BMC Neurol 2015;15:253.

12. Wolters FJ, Li L, Gutnikov SA, et al. Medical attention seeking after transient ischemic attack and minor stroke before and after the UK Face, Arm, Speech, Time (FAST) public education campaign: results from the Oxford vascular study. JAMA Neurol 2018;75:1225-33.

13. Edwards JD, Kapral MK, Fang J, et al. Long-term morbidity and mortality in patients without early complications after stroke or transient ischemic attack. Can Med Assoc J 2017;189:E954-61.

14. Lovett JK, Coull AJ, Rothwell PM. Early risk of recurrence by subtype of ischemic stroke in population-based incidence studies. Neurology 2004;62:569-73.

15. Giles MF, Rothwell PM. Risk of stroke early after transient ischaemic attack: a systematic review and meta-analysis. Lancet Neurol 2007;6:1063-72.

16. Wang $Y$, Zhao $X$, Jiang $Y$, et al. Prevalence, knowledge, and treatment of transient ischemic attacks in China. Neurology 2015;84:2354-61.

17. Bhattacharya $P$, Khanal D, Madhavan R, et al. Why do ischemic stroke and transient ischemic attack patients get readmitted? $J$ Neurol Sci 2011;307:50-4.

18. Kilkenny MF, Dewey HM, Sundararajan V, et al. Readmissions after stroke: linked data from the Australian Stroke Clinical Registry and hospital databases. Med J Aust 2015;203:102-6.

19. Olson DM, Cox M, Pan W, et al. Death and rehospitalization after transient ischemic attack or acute ischemic stroke: one-year outcomes from the adherence evaluation of acute ischemic strokelongitudinal registry. J Stroke Cerebrovasc Dis 2013;22:e181-8.

20. Jackova J, Sedova P, Brown RD, et al. The High Frequency of Guideline-Approved and Guideline-Disapproved Medication Use in Stroke and Transient Ischemic Attack. J Stroke Cerebrovasc Dis 2016;25:2688-93.

21. Morris S, Hunter RM, Ramsay Al, et al. Impact of centralising acute stroke services in English metropolitan areas on mortality and length of hospital stay: difference-in-differences analysis. BMJ 2014;349:g4757.

22. lacus SM, King G, Porro G. Multivariate Matching Methods That Are Monotonic Imbalance Bounding. J Am Stat Assoc 2011;106:345-61.

23. lacus SM, King G, Porro G. Causal Inference without Balance Checking: Coarsened Exact Matching. Political Analysis 2012;20:1-24.

24. Quan $\mathrm{H}$, Sundararajan $\mathrm{V}$, Halfon $\mathrm{P}$, et al. Coding algorithms for defining comorbidities in ICD-9-CM and ICD-10 administrative data. Med Care 2005;43:1130-9.

25. Prescott HC, Langa KM, Liu V, et al. Increased 1-year healthcare use in survivors of severe sepsis. Am J Respir Crit Care Med 2014;190:62-9.

26. Blackwell M, lacus S, King G, et al. Cem: Coarsened Exact Matching in Stata. Stata J 2009;9:524-46.

27. King M, Essick C, Bearman P, et al. Medical school gift restriction policies and physician prescribing of newly marketed psychotropic medications: difference-in-differences analysis. BMJ 2013;346:f264.

28. Tabuchi T, Murayama H, Hoshino T, et al. An Out-of-Pocket Cost Removal Intervention on Fecal Occult Blood Test Attendance. Am J Prev Med 2017;53:e51-e62.

29. Zhong W, Geng N, Wang P, et al. Prevalence, causes and risk factors of hospital readmissions after acute stroke and transient ischemic attack: a systematic review and meta-analysis. Neurol Sci 2016;37:1195-202.

30. Johansen HL, Wielgosz AT, Nguyen K, et al. Incidence, comorbidity, case fatality and readmission of hospitalized stroke patients in Canada. Can J Cardiol 2006;22:65-71.

31. Lee HC, Chang KC, Huang YC, et al. Readmission, mortality, and first-year medical costs after stroke. J Chin Med Assoc 2013;76:703-14. 
32. Tseng MC, Lin HJ. Readmission after hospitalization for stroke in Taiwan: results from a national sample. J Neurol Sci 2009;284:52-5.

33. Shih T, Nicholas LH, Thumma JR, et al. Does pay-for-performance improve surgical outcomes? An evaluation of phase 2 of the Premier Hospital Quality Incentive Demonstration. Ann Surg 2014;259:677-81.

34. Rothwell PM, Algra A, Chen Z, et al. Effects of aspirin on risk and severity of early recurrent stroke after transient ischaemic attack and ischaemic stroke: time-course analysis of randomised trials. Lancet 2016;388:365-75.

35. Luengo-Fernandez R, Gray AM, Rothwell PM. Effect of urgent treatment for transient ischaemic attack and minor stroke on disability and hospital costs (EXPRESS study): a prospective population-based sequential comparison. Lancet Neurol 2009;8:235-43.

36. Mavaddat N, Savva GM, Lasserson DS, et al. Transient neurological symptoms in the older population: report of a prospective cohort study--the Medical Research Council Cognitive Function and Ageing Study (CFAS). BMJ Open 2013;3:e003195.

37. Liu M, Zhang Q, Lu M, Ms L, et al. Rural and urban disparity in health services utilization in China. Med Care 2007;45:767-74.

38. Cheng L, Liu H, Zhang Y, et al. The impact of health insurance on health outcomes and spending of the elderly: evidence from China's New Cooperative Medical Scheme. Health Econ 2015;24:672-91.
39. Han L, Zhou W, Li W, Wf L, et al. Urbanization strategy and environmental changes: an insight with relationship between population change and fine particulate pollution. Sci Total Environ 2018;642:789-99.

40. Zhang X, Dupre ME, Qiu L, et al. Urban-rural differences in the association between access to healthcare and health outcomes among older adults in China. BMC Geriatr 2017;17:151.

41. Persson J, Holmegaard L, Karlberg I, et al. Spouses of Stroke Survivors Report Reduced Health-Related Quality of Life Even in Long-Term Follow-Up: Results From Sahlgrenska Academy Study on Ischemic Stroke. Stroke 2015;46:2584-90.

42. Gavish R, Levy A, Dekel OK, et al. The Association Between Hospita Readmission and Pulmonologist Follow-up Visits in Patients With COPD. Chest 2015;148:375-81.

43. Neuman MI, Hall M, Gay JC, et al. Readmissions among children previously hospitalized with pneumonia. Pediatrics 2014;134:100-9.

44. Kokotailo RA, Hill MD. Coding of stroke and stroke risk factors using international classification of diseases, revisions 9 and 10. Stroke 2005;36:1776-81.

45. Cadilhac DA, Kilkenny MF, Levi CR, et al. Risk-adjusted hospital mortality rates for stroke: evidence from the Australian Stroke Clinica Registry (AuSCR). Med J Aust 2017;206:345-50. 